Original Research Paper

\title{
From Structural Colors to Super-Hydrophobicity and Achromatic Transparent Protective Coatings: Ion Plating Plasma Assisted $\mathrm{TiO}_{2}$ and $\mathrm{SiO}_{2}$ Nano-Film Deposition
}

\author{
${ }^{1}$ Raffaella Aversa, ${ }^{1}$ Valeria Perrotta, ${ }^{2}$ Relly Victoria V. Petrescu, \\ ${ }^{3}$ Misiano Carlo, ${ }^{2}$ Florian Ion T. Petrescu and ${ }^{1}$ Antonio Apicella \\ ${ }^{I}$ Advanced Material Lab, \\ Department of Architecture and Industrial Design, Second University of Naples, Aversa (CE) Italy \\ ${ }^{2}$ ARoTMM-IFToMM, Bucharest Polytechnic University, Bucharest, (CE) Romania \\ ${ }^{3}$ Romana film Sottili Srl, (CE), Italy
}

Article history

Received: 16-11-2016

Revised: 21-11-2016

Accepted: 23-11-2016

Corresponding Author:

Apicella Antonio

Advanced Material Lab, Department of Architecture and Industrial Design Second

University of Naples, Aversa

(CE) Italy

Email: Antonio.apicella@unina2.it

\begin{abstract}
The implementation of the Ion Plating Plasma Assisted technology in the area of surface functionalization for structural color and relic preservation applications is presented. Interferometric structural colors on irregular bumped Titanium surfaces and transparent and achromatic nano films on ancient ceramic artifact have been investigated. Titanium metal and ceramic supports have been utilized for the surface functionalization tests: A metallic electron beam additive manufactured Titanium component and an ancient tile of the XIX century, which was characterized by strong chromatic valence and by a mixed porous and glazed surfaces, have been considered. A reactive magnetron sputtering Ion Plating Plasma Assisted apparatus operating in Argon or Oxygen atmospheres for $\mathrm{TiO}_{2}$ and $\mathrm{SiO}_{2}$ deposition has been utilized. Preliminary tests with two plasma treatments were carried out for optimal processing conditions definition. $\mathrm{TiO}_{2}$ nano-film deposition on irregular Ti surfaces has generated light direction depending color-changing surfaces while good achromatic and transparent coatings were obtained by using $\mathrm{SiO}_{2}$ coating. The high processing flexibility of the Ion plating technology is discussed. The $\mathrm{SiO}_{2}$ IPPA surfaces treatment resulted more convenient for restorative and preservation ancient historical tile was used to finally test the optimized process with Ion Beam Electron Microscopy, which was carried out on the tile porous structure, confirmed the high flexibility and efficiency of the innovative IPPA technology.
\end{abstract}

Keywords: Ion Plating Plasma Assisted, Structural Colors, Cultural Heritage Preservation, Biomedical Application

\section{Introduction}

Not all colors in nature come from the material inherent chemical structure coloring. There are several physical phenomena that drive surface coloring by light wavelength interference or diffraction. The former one, which is often reported as iridescence or structural coloring, has been investigated since late 17 th century. Hooke (2003) speculates that alternate solid and air thin layers strongly reflected the light. Newton (1730) in Opticks that the colors of the iridescent peacock arose from the thinness of the transparent part of the feathers.
Further scientific advancement on genesis of structural colors has been interpreted in terms of electromagnetic theory by Maxwell (Chrystal, 1873) and by experimental studies of electromagnetic waves by Hertz (1884). Rayleigh $(1917 ; 1919)$ used the electromagnetic theory to derive the concept to express the reflection properties from a regularly stratified medium. This theory was the base to explain the nature of the colors of crystals, feather and beetles and butterflies (Kwok et al., 1997), which varied with the incidence of light direction, were not inherent colors but structural colors (Aronov and Rosenman, 2007). 
The study and understanding of natural structural coloring of insects and bird feathers is being attracting great interest because their use could find applications in many commercial fields related to product aesthetic, such metal coloring, dental implants, cosmetics and textiles.

A complete understanding of these structures was first achieved with the use of the electron microscopy and more recently, by the ion double beam microscopy and Atomic force microscopy that enabled us the vision of the micro and nano structure morphology of natural born structural color generating systems. These studies (Kinoshita and Yoshioka, 2005) have clarified that coloring are due to the presence of specific micro and nanostructures, which cannot be easily realized by the know present nanotechnologies that, then, need to be further technologically developed.

Essentially, these structural coloring originate themselves from optical phenomena driven by single or multilayer thin-film interference, photonic nano-crystals (quantum dots), light scattering and a diffraction grating effect. Living creatures have developed in the course of their evolution various micro-structural designs that concurrently fulfill high reflectivity for specific wavelength ranges while generating wide angular range diffusive light. Even if these two physical characteristics seem to optically oppose each other, when combined in appropriate combinations where morphological regularity and irregularity assume specific configurations, the resulting system is able to filters and enhances specific colors.

In this paper, we present exploratory investigations that combine simple and complex surface morphologies to explain some fundamental optical properties underlying the structural colors.

The simplest single thin film transparent coating has been investigated for an application in the cultural heritage preservation, while complex surface morphologies obtained by plasma assisted thin film deposition have been investigated to obtain changing color surface.

Preserving cultural heritage is a general concern and the use of not-invasive techniques to protect and conserve ancient materials is an important challenge. Serious surface deterioration effects in environmentally exposed ancient pavements glazed ceramic tiles arise both from mechanical abrasion due to the tourist passage and for the development of micro-organisms (algae/fungi) within the pore system. Exfoliation often follows from biodegradation processes that are particularly harmful since leading to the decorated glaze severe damage (Silva et al., 2013; Ventolà et al., 2014). The use of traditional organic resin spray coating or wax treatments may partially reduce the problem but they seriously alter the tiles chromatic aesthetics. Cleaning and conservation of these ancient cultural handcrafts, therefore, need surface process applying innovative noninvasive techniques. The present work is intended as a contribution to implement new technologies for the deposition functional coatings in the aea of biomedicine, design and protective coatings preventing degradation of ancient tiles.

The development in protection treatment in which the introduction of an IPPA process by thermal source (Misiano, 2001; Mattox, 1964) seems to be very promising with respect to process using traditional organic polymeric coatings. The growing demand for multi-layered thin film deposition opens new applications for sputter technology. The deposition of highly strength oxide layers such as $\mathrm{SiO}_{2}$ and $\mathrm{TiO}_{2}$ used in this study, has turned out to be rather satisfactory innovation of great practical significance in several field of application. The use of such techniques in the area of aesthetic and historical heritage conservation is presented in this study.

Bioactive surface modification and aesthetic effect can be obtained by designing the system according to destructive and constructive interference reflections by choosing the correct refractive index transparent coating material, the thickness of the nanomeric coating and reflecting surface morphology.

Functional coatings for early bone growth can be combined to aesthetic appearance that may be needed in some chirurgical situations (aesthetic surgery on tooth implant replacement).

\section{Materials and Procedures}

\section{Materials}

Additive manufactured Titanium samples of complex structure and ceramic tiles have been utilized for the tests: two contemporaneous production tiles, all characterized by strong chromatic valence and by mixed porous tile and glazed surfaces and a component electro beam manufactured in a plant for metal prostheses production have been used in our study.

\section{Testing Procedures and Apparatus}

Ion Plating Plasma Assisted (IPPA)- two surface coatings procedures were tested and compared on the contemporaneous tile surfaces: Deposition of nanomeric Ion Plating Plasma Assisted protective films of a $\mathrm{TiO}_{2}$ and $\mathrm{SiO}_{2}$.

Sputtering is a PVD coating technology. Inert gas atoms are sent into a high vacuum environment at very low pressure (generally below 10 mTorr) where their atoms are ionized, creating a gas-like atmosphere, that will cause them to collide with the target (in our case Ti or Silicon Oxide).

These collisions enable the target atoms to eject toward the substrate to coat where they condense forming a thin-film. We can configure systems to support RF sputtering and different target sources (such as thermal or magnetron). 
The strong magnetic field created in the sputtering process leads the ions and electrons that are generated inthe plasma to be confined to a designated area close to the source target. This particular configuration generates more collisions between ions and neutral gas molecules creating more ions that could collide with the source target ejecting larger amounts of base coating material ( $\mathrm{Ti}$ or $\mathrm{SiO}_{\mathrm{x}}$ in our study). Due to the presence of a higher number of ions and of an electric field, this process allows a higher rate of deposition.

Magnetron sputtering assists reducing damage to the thin film because the magnetic field guides electrons to travel in specific routes, without hitting the substrate.

Owing to the higher energy of the ejected material from the target, magnetron-sputtering technology creates very dense coatings with increased adhesion.

Moreover, when a reactive gas such as Oxygen or Nitrogen is introduced into the sputtering chamber; reactions occur with these ionized gases to form oxide and nitride films. In reactive sputtering, the sputtered particles undergo a chemical reaction before coating the substrate and, then, the deposited coating has a composition different from the target material.

The two equipment used for Ion Plating Plasma Assisted deposition with magnetron sputtering target and thermal source were composed by a high-vacuum chamber containing sources of $\mathrm{Ti}$ for sputtering source and $\mathrm{SiO}_{\mathrm{x}}$ for thermal source attached to a magnetron sputter source, powered by a Direct Current (DC) as shown in Fig. 1. Sputtering source employing magnetron utilizes heavy electric and magnetic fields to confine the particles of the charged plasma near to the surface of the sputter target.

In the chamber, along with the samples, a gas mixture of Argon and Oxygen was introduced. The percentage of ionized depositing material (Titanium or $\mathrm{SiO}_{\mathrm{x}}$ particles) emitted by the DC powered magnetron sputtering or thermal sources, are accelerated by a negative bias produced by a Radiofrequency electric Field (RF) applied to the substrate holder. Such radiofrequency produces plasma in the processing atmosphere, which produce a ionization of the depositing materials and produces an ion bombardment of Argon and Oxygen on the growing film (Misiano 2001; Mattox, 1964; Misiano and Scandurra, 2006).

Ion Plating Plasma Assisted source by magnetron sputtering with Argon atmosphere Preliminary Vacuum $7,0 \times 10^{-5}$ mbar and an Argon atmosphere of $3,0 \times 10^{-3}$ mbar, $\mathrm{O}_{2}$ pressure $410^{-4}$ mbar was used to deposit $\mathrm{TiO}_{2}$ in the plant Balzer BA 710 (IPPARCUS), with a $\mathrm{Ti}$ target. The deposited Titanium Dioxide was characterized by a high Refractive Index of 2.4.

The second film deposition test was carried out from the heat source with $\mathrm{SiO}_{\mathrm{x}}$ : Argon atmosphere preliminary Vacuum $5,0 \times 10^{-5}$ mbar, Pressure $\mathrm{Ar}$ $3,0 \times 10^{-3}$ mbar, Pressure O2 $1.8 \times 10^{-4}$ mbar.

The $\mathrm{SiO}_{2}$ thin film deposition carried out at pressure of $\operatorname{Ar} 3,0 \times 10^{-3}$ mbar reached a deposition rate $3.5 \mathrm{~nm}$ $\mathrm{sec}^{-1}$ leading to a coating with an average thickness of $500 \mathrm{~nm}$, Radiofrequency was $24 \mathrm{~W}, 200 \mathrm{~V}$ bias with evaporation of $\mathrm{SiO}_{\mathrm{x}}$. The deposited Silicon dioxide showed a Refractive Index 1.46.

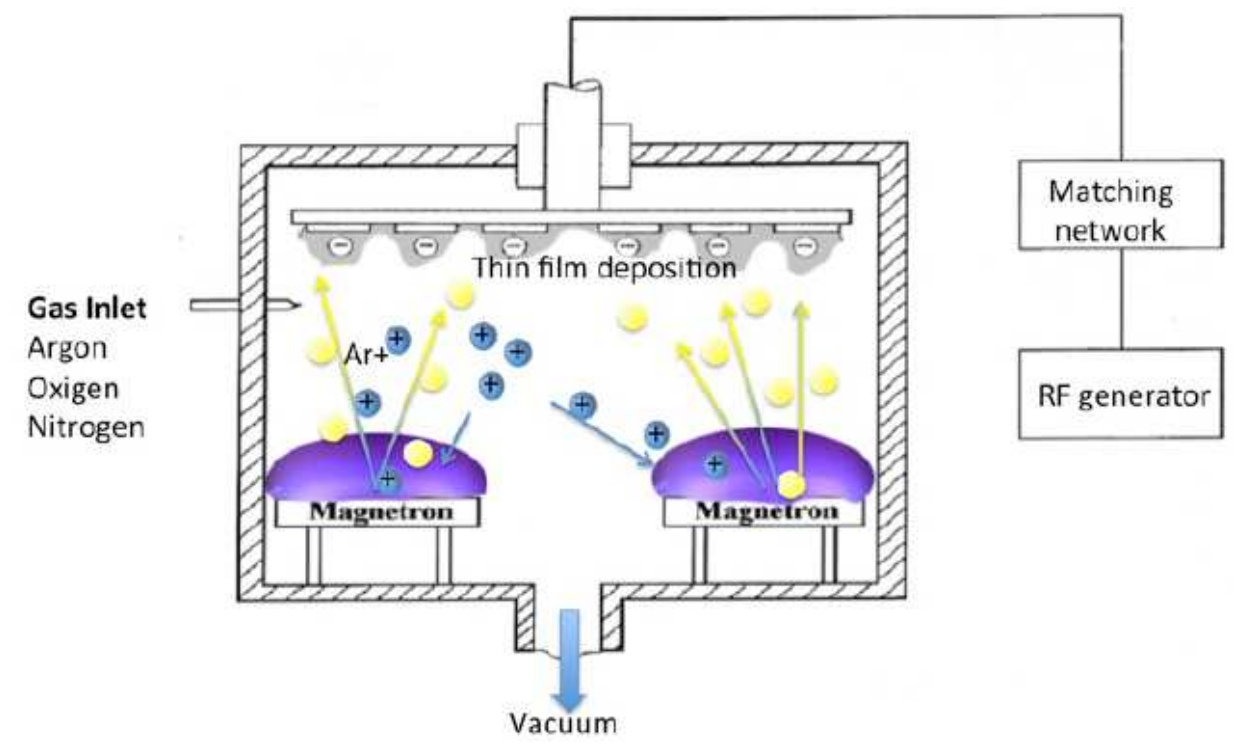

Fig. 1. Experimental set-up for Ion Plating Plasma Assisted (IPPA) from thermal and magnetron sources for transparent $\mathrm{SiO}_{2}$ and $\mathrm{TiO}_{2}$ deposition 


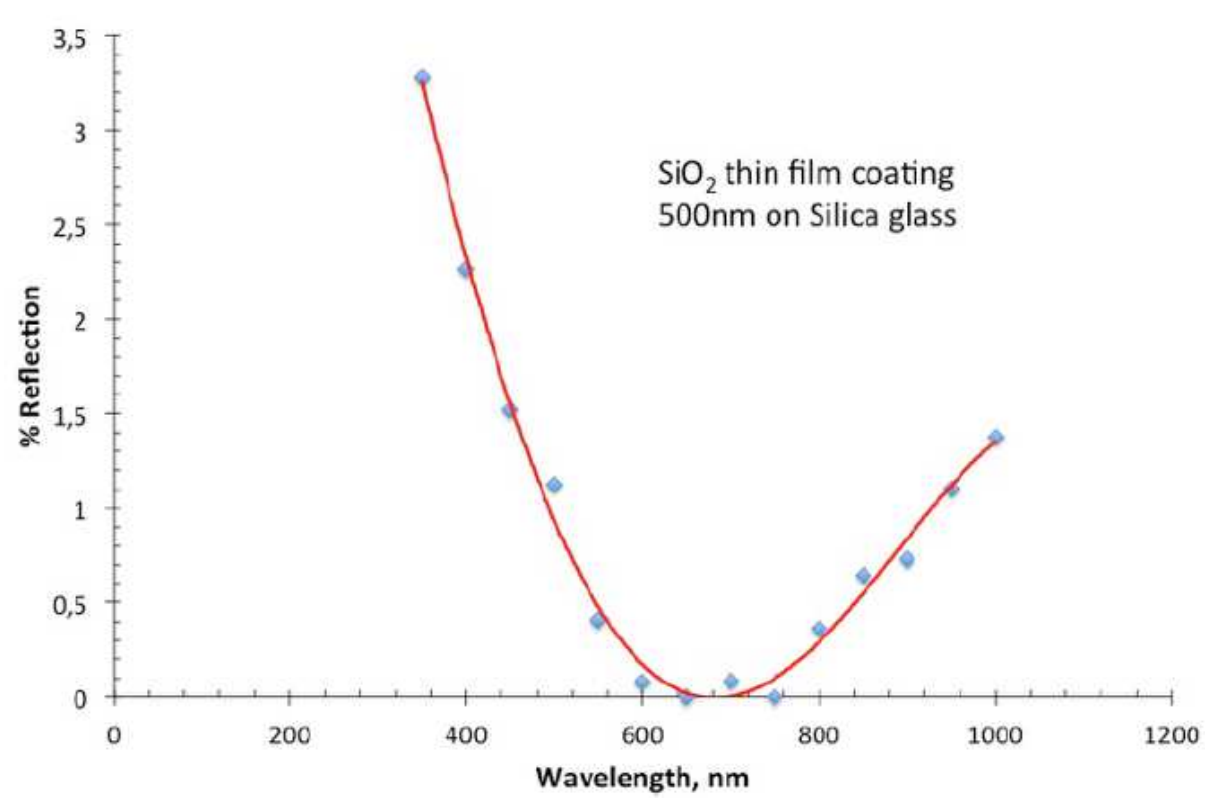

Fig. 2. Reflection \% for Ion Plating Plasma Assisted (IPPA) $\mathrm{SiO}_{2}$ protective thin film coating on silica glass

The coated samples were spectro-photo-metrically verified as reported in Fig. 2 where the \% reflection as a function of light radiation wavelength are reported for a $500 \mathrm{~nm} \mathrm{SiO}{ }_{2}$ coating on glass substrate.

\section{Results and Discussion}

\section{Protective Coating-TiO $\mathrm{TPPA}_{2}$ Coating on Ceramic Support}

The result of the IPPA treatment on a semi glazed tiles using $\mathrm{TiO}_{2}$ deposition is shown in Fig. 3 (left hand).

This treatment, even if it is able to guaranty a homogeneous and transparent film deposition, is characterized by an evident color change of the glazed surfaces. This is due to the high refractive index (2.4) that, for this specific thin film thickness $(400 \mathrm{~nm})$, produces greenish-pink color nuances due to the constructing interference reflection shown in Fig. 4. The porous surface is not altered by the presence of the thin film coating (about $400 \mathrm{~nm}$ ).

\section{Protective Coating-SiO ${ }_{2}$ IPPA Coating on Ceramic Support}

A second IPPA treatment using $\mathrm{SiO}_{2}$ is shown in Fig. 2 (left). This treatment is able to guaranty a homogeneous and achromatic transparent film deposition.

This behavior can be clarified the consideration to be done according to the physics of coating light transmission properties reported in Fig. 4.

The reflected and refracted light paths and intensities depend upon the incident light wavelength $\left(\lambda_{1}\right.$ and $\left.\lambda_{2}\right)$, coating and substrate refractive indices, thickness of the coating (L) and the angle of the incident light $\theta$.

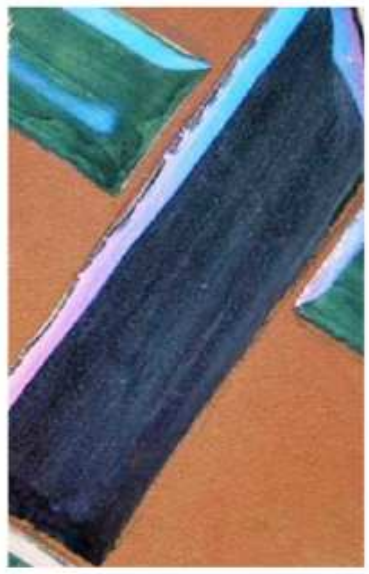

$\mathrm{TiO}_{2}$ thin film coating

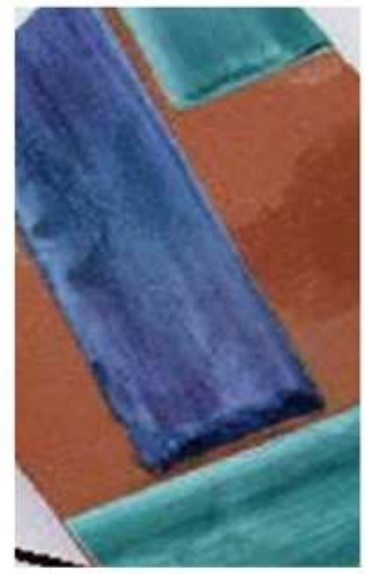

$\mathrm{SiO}_{2}$ thin film coating
Fig. 3. Ion Plating Plasma Assisted (IPPA) $\mathrm{TiO}_{2}$ (Left) and $\mathrm{SiO}_{2}$ (Right) protective thin film coating on ceramic tiles

As light passes through an uncoated glass substrate, approximately $4 \%$ will be reflected at each interface. This results in a total transmission of only $92 \%$ of the incident light.

Anti-reflection coatings are particularly important if the coated surface has to preserve its aesthetic quality.

The coating is designed so that the relative phase shift between the beam reflected at the upper and lower boundary of the thin film is $180^{\circ}$. Destructive interference between the two reflected beams $r_{1}$ and $r_{2}$ occurs, cancelling both beams before they exit the surface (upper part of Fig. 4). 

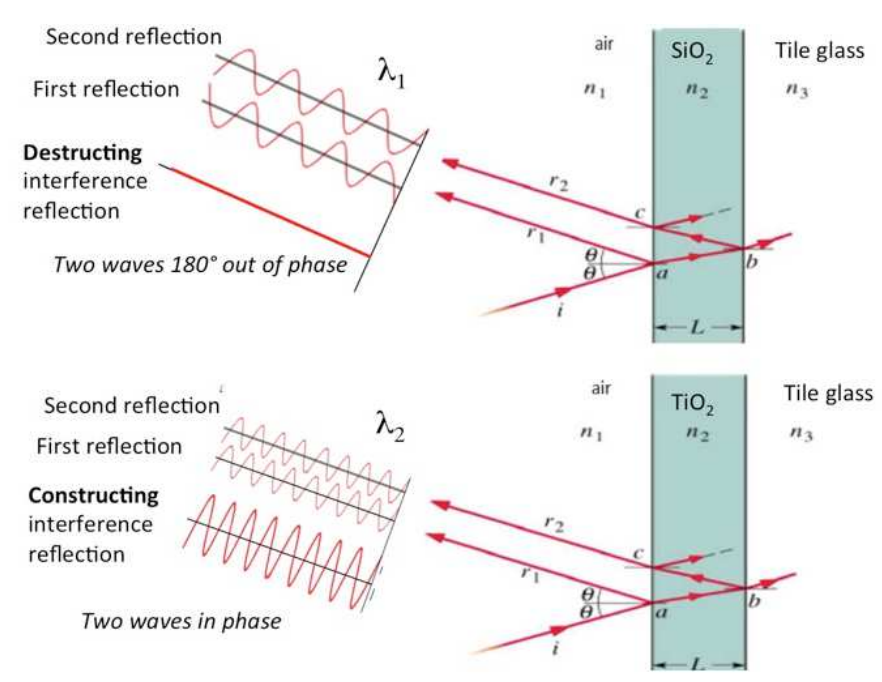

Fig. 4. Destructive and Constructing interference reflection effect in planar thin films of $\mathrm{SiO}_{2}$ (upper part) and $\mathrm{TiO}_{2}$ (lower part)

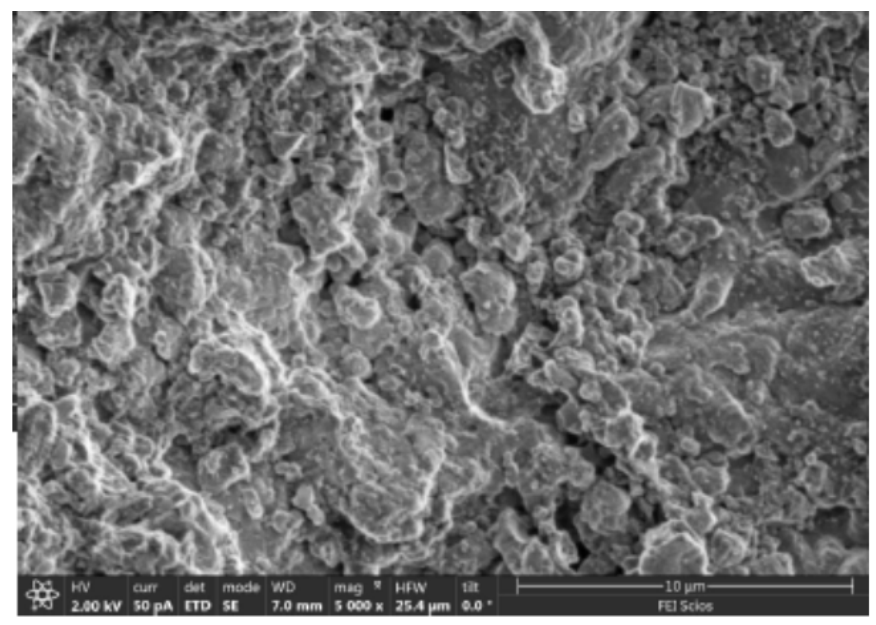

Fig. 5. Dual Beam FEI Microscopy of $\mathrm{SiO}_{2}$ coated surface of the unglazed part of the tile

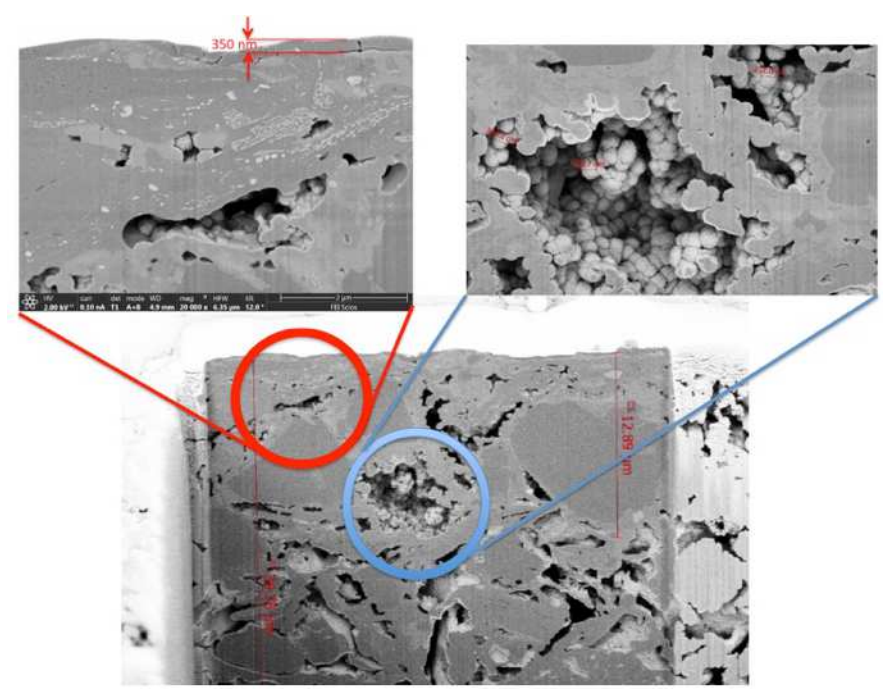

Fig. 6. Upper part-Details of Dual Beam FEI Microscopy of $\mathrm{SiO}_{2}$ coated porous tile. Bottom Ion Beam section of the porous ceramic tile 


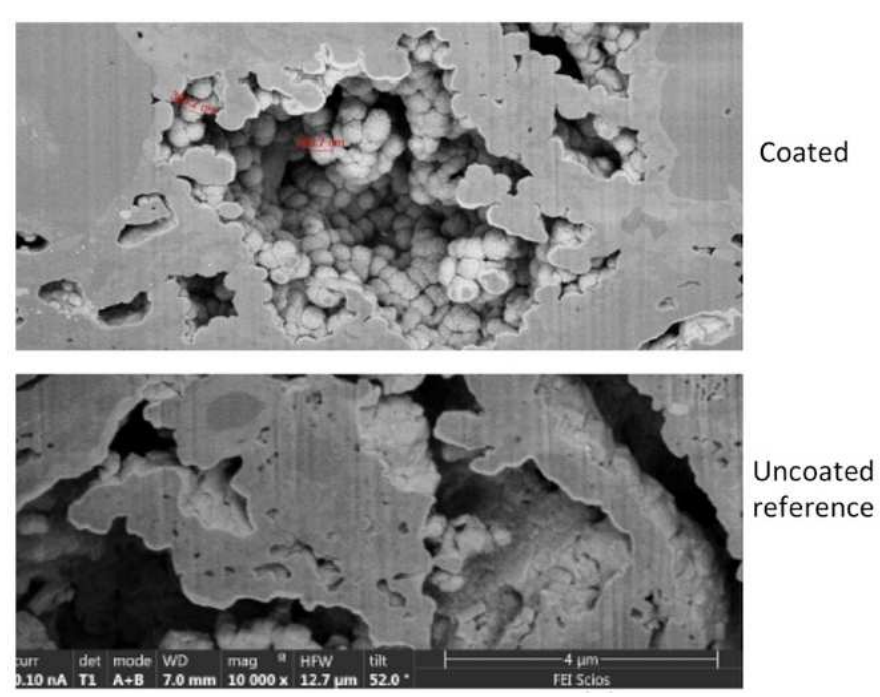

Fig. 7. Dual Beam FEI Microscopy of $\mathrm{SiO}_{2}$ coated porous tile. Bottom Ion Beam transverse section on the IPPA $\mathrm{SiO}_{2}$ coated porous tile (top) and of an uncoated reference tile

The optical thickness of the coating must be an odd number of quarter wavelengths $(\lambda / 4$, where $\lambda$ is the design wavelength or wavelength being optimized for peak performance), in order to achieve the desired path difference of one half wavelength between the reflected beams, which leads to their cancellation.

The image of the unglazed tile surface after $\mathrm{SiO}_{2}$ Ion assisted plasma coating is shown in Fig. 5 .

The surface shows the presence of sub-micron pores that have not been obstructed by the plasma treatment. The aesthetics of the porous surface with no glaze is not altered by the presence of the $\mathrm{SiO}_{2}$ thin film coating, which has been evaluated from SEM microscopy to be of about 350-400 nm).

Figure 6 reports the Ion beam transverse section obtained in a FEI, Scios DualBeam SEM microscope of the unglazed tile surface treated plasma coated of $\mathrm{SiO}_{2}$.

The transverse section (bottom part of Fig. 6) of the unglazed tile shows a highly porous internal structure with irregular porous size of $0.5-3.0 \mu \mathrm{m}$ (Fig. 6 and 7). Inside the pores it has been noticed the presence of bulb shaped formations of $350-400 \mathrm{~nm}$ that are not visible in the pores of the reference tile samples no treated with Ion Plasma assisted deposition (bottom part of Fig. 7).

Figure 7 , in fact, reports the micrograph of the Ion beam transverse sections of a plasma assisted IPPA coated (top) and of the uncoated reference (bottom) of an unglazed porous tile.

The external tile surface appears uniformly coated with a constant thickness layer of about 350-400 nm (detail upper left in Fig. 7). It can be inferred, hence, that the coating of the tile does not interest the surface only, but it is also penetrated in the internal cavities of the pores.

However, even if the pores show a reduced lumen, they are not obstructed by the plasma deposition.
It could be expected that the presence of the coating treatment inside the pores could guaranty an even more effective protection for liquid water penetration in the ceramic tile porosity reducing the risk of fungi or mildews unwanted development. The spheroidal $\mathrm{SiO}_{2}$ crystal irregular growth covers the entire pore wall surface creating a continuous but rough layer.

The effect of roughness on the contact angle and surface wettability is relevant (Aronov and Rosenman, 2007; Meiron et al., 2004; Nosonovsky and Bhushan, 2008; Patankar, 2003) and several example can be found in nature, such as the Lotus leaves, where surface morphology cooperates with surface tensions to generate "superhydrofobicity" (Soeno et al., 2004; Spori et al., 2008).

The roughness on silicon dioxide can suggest that the rougher is the surface the more it will be able to repel water. By controlling the surface structure of a substrate we can decrease wettability and improve it hydrophobic. Soeno et al. (2004) points out that rougher $\mathrm{SiO}_{2}$ coated surfaces repel water more intensely. This occurrence can be justified in terms of apparent contact angles, surface morphologies and air trapping ability of the irregular surfaces.

Cassie model of wetting has been used (Yoshimitsu et al., 2002; Kwok et al., 1997; Letellier et al., 2007; Vukusic et al., 1999) to describe the effect of roughness on apparent contact angle variations:

$\cos \theta^{\prime}=f(\cos \theta+1)-1$

where, $\theta^{\prime}$ is the apparent contact angle of the rough surface, $\mathrm{f}$ is the fractional area of the solid over the gas phases contact with the wetting liquid, namely the ratio between the wetted area and the overall surface area and $\theta$ is the contact angle on a flat surface, namely the 
Young contact angle (Spori et al., 2008; Kinoshita et al., 2002). This model, even when the true contact angle of a liquid on a smooth surface is less than $90^{\circ}$, predicts that the contact angle can be enhanced (this is due to the trapped air bubbles that decrease the solid surface wet fraction).

The spheroidal shape of the particles creates a high new surface area that can be evaluated from their mean diameter (330 nm from Fig. 6 top part). The dense packing of the spheroids for surface of a pore cavity of the same dimension (lower part of Fig. 6) leads to evaluate a roughness factor ranging from 4.5 to 6.0 and an increased area for air trapping.

According to equation 1 , a true silica-water contact angle of $15^{\circ}-20^{\circ}$ and a fraction of air trapped on the plasma generated irregular $\mathrm{SiO}_{2}$ surface inside the pore cavities of 0.5 could lead to an increase of the apparent contact angle that overcome $90^{\circ}$ (hydrophobicity).

A plasma ion assisted treatment using $\mathrm{SiO}_{2}$ coating on the porous ceramic substrate, is producing, therefore, an external flat surface characterized by a good wettability and by irregular surfaces inside the pore cavities, which are characterized by a hydrophobicity, that could avoid liquid water penetration and stagnation in the pores.

By choosing a deposited film material with higher contact angle (such as the $\mathrm{TiO}_{2}$ or other Fluorated substances, super-hydrophobic surfaces may be generated.

\section{Structural Colors-TiO 2 IPPA Coating on Metal Ti Support}

IPPA $\mathrm{TiO}_{2}$ deposition on a metallic support obtained by additive manufacturing technology employing $\mathrm{Ti}$ powder has been carried out in the same experimental condition utilized for the ceramic support.

The use of a metallic support will increase the percentage of light that will be reflected from the object. This occurrence will exalt the effect of the destructing and constructing interference reflection in the $\mathrm{TiO}_{2}$ coated system. It has previously discussed as the highly refracting (2.4 Refractive Index) Titanium Oxide coating layer could favor this phenomenon creating coloring interference effect in the flat surface coating of glazed ceramic support (Fig. 3).

In the case of a highly reflecting metal surface and concurrent multiple reflections, this effect of color "filtering" and "iridescence" of the treated surfaces is exalted. The iridescence effect will depend on the morphology of the surface, refractive index of the transparent coating and its thickness. The more the surface is irregular and bumbed, the higher will be the number of internal reflections and, therefore, the intensity of the colour filtering and iridescence effect (structural colors).

Figure 8 shows this iridescent effect on a complex shape $\mathrm{Ti}$ object obtained by electron beam additive manufacturing from Ti powder.

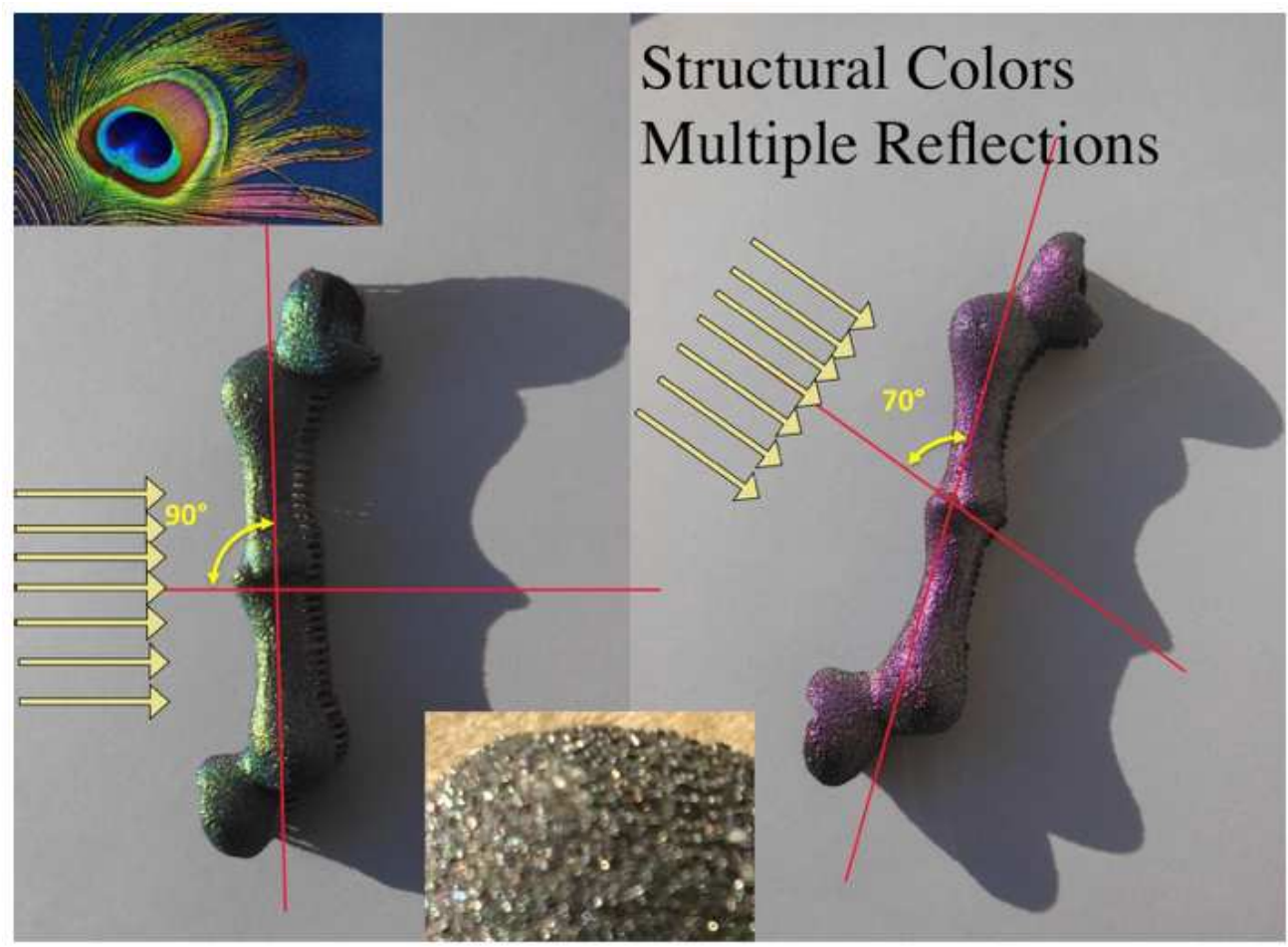

Fig. 8. Iridescence on the IPPA $\mathrm{TiO}_{2}$ coated irregular bumped surface Ti component 
A detail of the irregular and bumped surface of the object is shown in the central lower part of Fig. 8 .

The change of $20^{\circ}$ in the hitting sunlight direction causes a drastic change of the visualized color.

The gold-green color of the sample hit by the sunlight at $90^{\circ}$ turns to a vivid purple when the sunlight hits the object with an angle of $70^{\circ}$. The structural colors generated by the coating with $\mathrm{TiO}_{2}$ are similar to those observed for the peacock feathers (upper left of Fig. 7) and other birds.

This effect can be related to the physical phenomenon described in Fig. 4 (bottom) in the case of multiple reflections.

It has been described by Kinoshita and Yoshioka, (2005) as multiple reflections in regular or irregular surface morphology can create color enhancing or cancelling effects.

\section{Conclusion}

Due to recent advances on plasma deposition technology (Misiano, 2002; Misiano and Scandura, 2006; Annunziata et al., 2006), it has been possible to define innovative efficient treatment for biomedical applications and for the preservation and protection of historical ancient relics of $\mathrm{SiO}_{2}$ and $\mathrm{TiO}_{2}$ deposited by IPPA process.

Ion Plating Plasma Assisted (IPPA) $\mathrm{TiO}_{2}$ and $\mathrm{SiO}_{2}$ nano-film deposition could preferentially induce a wide range of surface effects going from structural coloring to acrhomatic transparent protective coatings or superhydrofobicity depending on the deposited material surface characteristics and on the substrate surface morphologies.

Such treatment can be useful in several fields that include biomedical application as well as aesthetic dental surgery (Annunziata et al., 2006) or relic preservation.

The presence of the coating treatment inside the pores could guaranty an even more effective protection for liquid water penetration in the ceramic tile porosity reducing the risk of fungi or mildews unwanted development. Nevertheless, the external surface maintains a good wettability necessary for the cleaning operation (the wet surface will not appear "oleaginous").

The same technology has been demonstrated to be able to induce very strong structural color and iridescence effects.

It has been confirmed by our investigation on structural colors obtained by $\mathrm{TiO}_{2}$ coating, thatmultiple reflections in regular or irregular surface morphology, as described by Kinoshita and Yoshioka (2005), can create color enhancing or cancelling effects.

In particular, it has been experimentally demonstrated that the presence of an irregular bumped surface (such as those obtained by additive manufacturing) could enhance structural color generation.

\section{Acknowledgement}

The Authors acknowledge Dr Francesco Tatti (FEI Company Application Specialist SEM-SDB) for its contribut in the preparation of SEM analyses.

\section{Author's Contributions}

All the authors contributed equally to prepare, develop and carry out this work.

\section{Ethics}

This article is original. Author declares that are not ethical issues that may arise after the publication of this manuscript.

\section{References}

Annunziata, M., R. Aversa, A. Apicella, A. Annunziata and D. Apicella et al., 2006. In vitro biological response to a light-cured composite when used for cementation of composite inlays. Dental Mater., 22: 1081-1085. DOI: 10.1016/j.dental.2005.08.009

Aronov, D. and G. Rosenman, 2007. Wettability study of modified silicon dioxide surface using environmental scanning electron microscopy. J. Applied Phys., 101: 084901-084901.

DOI: $10.1063 / 1.2721945$

Chrystal, G., 1873. A treatise on electricity and magnetism. Nature, 7: 478-480.

DOI: $10.1038 / 007478 \mathrm{a} 0$

Hertz, 1884. Wiedemann Annalen, 23: 84-103.

Hooke, R., 2003. Micrographia, or, Some Physiological Descriptions of Minute Bodies made by Magnifying Glasses: With Observations and Inquiries Thereupon. 1st Edn., Courier Corporation, Mineola, ISBN-10: 0486495647, pp: 273.

Kinoshita, S. and S. Yoshioka, 2005. Structural colors in nature: The role of regularity and irregularity in the structure, ChemPhysChem, 6: 1442-1459.

DOI: $10.1002 /$ cphc. 200500007

Kinoshita, S., S. Yoshioka and K. Kawagoe, 2002. Mechanisms of structural colour in the Morpho butterfly: Cooperation of regularity and irregularity in an iridescent scale. Proc. R. Soc. Lond. B, 269: 1417-1421. DOI: 10.1098/rspb.2002.2019

Kwok, D.Y., T. Gietzelt, K. Grundke, H.J. Jacobasch and A.W. Neumann, 1997. Contact angle measurements and contact angle interpretation. 1 . Contact angle measurements by axisymmetric drop shape analysis and a goniometer sessile drop technique. Langmuir, 13: 2880-2894.

DOI: $10.1021 / 1 a 9608021$ 
Letellier, P., A. Mayaffre and M. Turmine, 2007. Drop size effect on contact angle explained by nonextensive thermodynamics. Young's equation revisited. J. Colloid Interface Sci., 314: 604-614. DOI: $10.1016 /$ j.jcis.2007.05.085

Mattox, D.M., 1964. Electrochem. Technol., 2: 295-295.

Meiron, T.S., A. Marmur and I. Saguy, 2004. Contact angle measurement on rough surfaces. J. Colloid Interface Sci., 274: 637-644. DOI: $10.1016 /$ j.jcis.2004.02.036

Misiano, C. and R. Scandurra, 2006. Osteointegration process for surgical prostheses. U.S. Patent, n. 20080237033 .

Misiano, C., 2001. Cost effective high performance coatings by ion plating. Society of Vacuum Coaters, Albuquerque.

Newton, S.I., 1730. Opticks: Or, A Treatise of the Reflections, Refractions, Inflections and Colours of Light. 1st Edn., reprinted 1952, Dover Publications, New York, pp: 406.

Nosonovsky, M. and B. Bhushan, 2008. Cassie-Wenzel Wetting Regime Transition. In: Multiscale Dissipative Mechanisms and Hierarchical Surfaces, Friction, Superhydrophobicity and Biomimetics, Springer, Berlin Heidelberg New York, pp: 153-167.

Patankar, N.A., 2003. On the modeling of hydrophobic contact angles on rough surfaces. Langmuir, 19: 1249-1253. DOI: $10.1021 / \mathrm{la026612+}$

Rayleigh, L., 1917. Roy. Soc. Proc. A93: 565-577.

Rayleigh, L., 1919. Philos. Mag., 37: 98-121.
Silva, T.P., M.O. Figueiredo and M.I. Prudêncio, 2013. Ascertaining the degradation state of ceramic tiles: A preliminary non-destructive step in view of conservation treatments. Applied Clay Sci., 82: 101-105. DOI: 10.1016/j.clay.2013.06.013

Soeno, T., K. Inokuchi and S. Shiratori, 2004. Ultrawater-repellent surface: Fabrication of complicated structure of $\mathrm{SiO}_{2}$ nanoparticles by electrostatic selfassembled films. Applied Surface Sci., 237: 539-543. DOI: 10.1016/j.apsusc.2004.06.041

Spori, D.M., T. Drobek, S. Zürcher, M. Ochsner and C. Sprecher et al., 2008. Beyond the lotus effect: Roughness influences on wetting over a wide surface-energy range. Langmuir, 24: 5411-5417. DOI: $10.1021 /$ la800215r

Ventolà, L., A. Cordoba, M. Vendrell-Saz, P. Giraldez and R. Vilardell et al., 2014. Decorated ceramic tiles used in Catalan modernist architecture (c.1870 to c.1925): Composition, decay and conservation. Construct. Build. Mater., 51: 249-257. DOI: 10.1016/j.conbuildmat.2013.10.083

Vukusic, P., J.R. Sambles, C.R. Lawrence and R.J. Wootton, 1999. Quantified interference and diffraction in single Morpho butterfly scales. Proc. R. Soc. Lond. B, 266: 1403-1411. DOI: $10.1098 /$ rspb.1999.0794

Yoshimitsu, Z., A. Nakajima, T. Watanabe and K. Hashimoto, 2002. Effects of surface structure on the hydrophobicity and sliding behavior of water droplets. Langmuir, 18: 5818-5822.

DOI: $10.1021 / \mathrm{la} 020088 \mathrm{p}$ 